\title{
Modélisation du régime annuel des petites rivières en vue d'installation de microcentrales hydroélectriques
}

\author{
Rija N. RANDRIANARIVONY ${ }^{1 *}$, Philippe LAURET ${ }^{2}$, Zely A. \\ RANDRIAMANANTANY ${ }^{1}$ ef Jean Claude GATINA ${ }^{2}$
}

'IME - Institut pour la Maîtrise de l'Energie, Université d'Antananarivo BP 566, Madagascar

${ }^{2}$ LPBS - Laboratoire de Physique des Bâtiments et des Systèmes, Université de La Réunion, 40 Av de Soweto, 97410 Saint Pierre

*Correspondance,courriel : nirinarija@yahoo.fr

\section{Résumé}

L'exploitation de l'Energie Nouvelle et Renouvelable est une priorité pour la mise en œuvre de la politique d'Electrification Rurale Décentralisée (ERD) de Madagascar. Le haut plateau et le versant est de l'île regorgent de potentialité pour l'installation de Micro et Pico Centrale hydroélectrique (MCH). Malheureusement le manque de données hydrologiques pénalise le développement de ce choix. La modélisation des régimes annuels des petits cours d'eau est alors nécessaire pour permettre la fiabilité des études de faisabilité relativement courte pour la taille de ces centrales. Les bases de données de l'Autorité pour la Protection contre les Inondations de la Plaine d'Antananarivo (APIPA) sont utilisées pendant la modélisation avec les Réseaux de Neurones Artificiels (RNA) de type Perceptron Multicouche. Les modèles retenus ont donné des résultats très exploitables et ont montré de polyvalence pour les petits bassins similaires.

Mots-clés : réseaux de neurones artificiels, modèles pluies-débits, régime annuel, petits bassins, microcentrale hydroélectrique

\section{Abstract}

Modelling of the annual mode of the small rivers for Micro Hydroelectric Power Station

The exploitation of New and Renewable Energy is a priority for the setting in work of the policy of Decentralized Rural Electrification (DRE) of Madagascar. The high 
plateau and the East slope of the island abound in potentiality for the installation of Micro and Pico hydroelectric power station (MCH). Unfortunately the lack of hydrological data penalizes the development of this choice. The modelling of the annual modes of the small rivers is then necessary to allow the reliability of the feasibility studies relatively short for the size of these power stations. The data bases of the Authority for Protection against the Floods of the Plain of Antananarivo (APIPA) are used during modelling with the Artificial Neural Network (RNA) of Multilayer Perceptron type. The models selected gave very useable results and showed versatility for the small similar basins.

Keywords : artificial neural Networks, rainfall-runoff models, annual Mode, small basins, Micro Hydroelectric Power Station

\section{Introduction}

Selon le Bilan national d'Energie, le bois est encore de loin la source la plus utilisée à Madagascar. Ceci est le reflet de la répartition géographique de la population et aussi le fait que l'énergie consommée est essentiellement domestique. Jusqu'à aujourd'hui seulement inférieur à $20 \%$ des ménages malgaches ont accès à l'électricité [1].

En tenant compte de l'émission des gaz à effet de serre dans le choix du système énergétique, les systèmes aux énergies nouvelles et renouvelables sont priorisés. Le Ministère de l'Energie et des Mines a identifié près de 300 sites dont la puissance annuelle combinée est d'environ $7.000 \mathrm{MW}$ pouvant produire $60.000 \mathrm{GWh}$ d'électricité. Or la puissance actuellement installée ne dépasse pas $250 \mathrm{MW}$ sur tout le territoire toutes sources confondues, soit seulement les $3 \%$ de la puissance potentielle. Le service de l'hydrologie a recensé à titre approximatif 30 sites d'une puissance au-delà de 50MW, 70 sites entre 1 et $5 \mathrm{MW}$, et 200 sites dans la plage de puissance potentielle inférieure à $100 \mathrm{~kW}$. Les sites de puissances plus faibles sont beaucoup plus nombreux et répondent aux besoins des communes ou localités rurales de Madagascar. La conférence de Lomé et celle de Katmandou en 1979 ont relevé la possibilité d'alimenter une centaine d'habitations avec 30kW [2].

Les MCH sont exploitées de façon durable, alors les données de longues durées sont les plus appréciées et sollicitées. L'inexistence de ces données nous amène à faire de la modélisation de ces petits cours d'eau.

Parmi les modèles pluies-débits, ceux de type boîte noire sont utilisés car ils peuvent relier la pluie au débit directement sans entrer en profondeur dans le processus 
hydrologique. Ceci a pour grand avantage de réduire au minimum les paramètres en entrée des modèles et ne pas accentuer le manque de données. Les réseaux de neurones sont très utilisés en hydrologie pendant la dernière décennie dans plusieurs objectifs [3-5]. Pour notre cas le RNA est utilisé pour constituer un modèle global de débits moyens journaliers interannuels des rivières avec très peu de données relevées sur site.

\section{Matériel et méthodes}

\section{2-1. Le réseau de neurones artificiels}

On trouve de différentes introductions sur les RNA dans la littérature, mais il est important de réitérer son inspiration par le neurone biologique [6]. Le neurone formel est représenté de la manière la plus commode à la Figure la. Les entrées du neurone sont les variables sur lesquelles opère le neurone, et la sortie est la valeur de la fonction d'activation $f$ souvent sigmoïde.

Les paramètres sont attachés aux entrées du neurone, la sortie du neurone est une fonction non linéaire d'une combinaison des entrées $\left\{x_{i}\right\}$ pondérées par les $\left\{w_{i}\right\}$ qui sont appelés sous le nom de "I poids $\|$. Le potentiel $v$ le plus fréquemment utilisé est la somme pondérée, à laquelle s'ajoute un terme constant ${ }^{w_{0}}$ ou ॥ biais ॥ : $v=w_{0}+\sum_{i=1}^{n} w_{i} x_{i}$

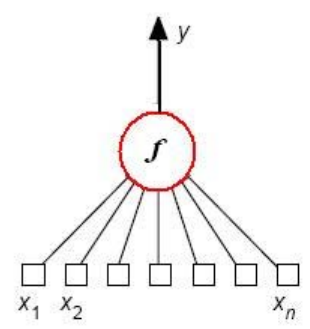

(a)

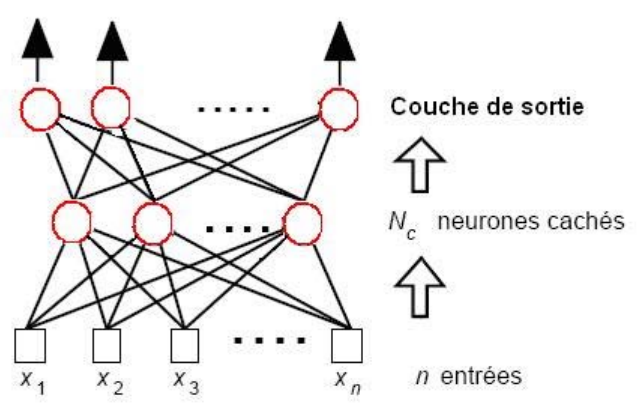

(b)

Figure 1 : Le neurone formel et le réseau multicouche

Un neurone réalise simplement une fonction non linéaire, paramétrée, de ses variables d'entrée. L'intérêt des neurones réside dans les propriétés qui résultent de 
leur association en réseaux, c'est-à-dire de la composition des fonctions non linéaires réalisées par chacun des neurones.

L'avantage de l'utilisation du RNA dans l'hydrologie est sa capacité à refléter la non linéarité inhérente à la relation pluie-débit sans entrer dans le processus (boîte noire). C'est une propriété intéressante pour ne considérer que les paramètres météorologiques d'autant plus que les paramètres hydrogéologiques sont quasiment inexistants pour notre cas. Les paramètres d'entrée pour tester les modèles sont groupés dans le Tableau $l$.

Tableau 1 :. Numérotation des paramètres d'entrée

\begin{tabular}{||l|l|l|l|l|l||}
\hline \hline $\mathrm{N}^{\circ}$ & 1 & 2 & 3 & 4 & 5 \\
\hline Paramètre & NumJ & $P^{j-1}$ & $H^{j-1}$ & $P^{j}$ & $H^{j}$ \\
\hline \hline
\end{tabular}

II existe plusieurs types de structures de réseaux de neurones. Pour modéliser le régime annuel des cours d'eau, nous choisissons la structure du Perceptron MultiCouche (PMC) (Figure Ib). L'architecture que nous avons adoptée contient une seule couche cachée de 7 neurones et une seule sortie représentée par la hauteur de la rivière au jour $j+1$. La sortie du réseau est alors indiquée par la relation (2):

$$
y=f\left(\sum_{j=1}^{N c} w_{k j} g\left(\sum_{i=1}^{n} w_{j i} x_{j}\right)\right)
$$

Initialement des réseaux à plusieurs couches cachées sont exploités mais il s'avère que le réseau à une seule couche est capable d'approximer toute fonction non linéaire à un degré de précision près [7].

Dans la relation (2) $g$ est la fonction d'activation sigmoïde de chaque neurone dans la couche cachée et $f$ celle du neurone de sortie linéaire.

Une phase d'apprentissage classique consiste à attribuer des valeurs initiales à l'ensemble des poids et biais, et à minimiser ensuite une fonction erreur quadratique $S / w$ )à l'aide de la base d'apprentissage et d'un algorithme d'apprentissage dérivé de méthode de rétropropagation de l'erreur [8].

$$
S(w)=\frac{1}{2} \sum_{i=1}^{N}\left\{y\left(x^{i} ; w\right)-t^{i}\right\}^{2}
$$


Avec $\mathrm{N}$ le nombre d'exemple dans la base d'apprentissage, $f$ la sortie désirée On présente au modèle neuronal un ensemble de valeurs en entrée, auxquelles sont associées des valeurs en sortie. Après plusieurs passages sur la base d'apprentissage, le réseau apprend la relation existant entre les variables d'entrée et la variable de sortie. Une fois l'apprentissage terminé, le réseau est, a priori, capable de généraliser, c'est-à-dire de donner des sorties correctes lorsque lui seront présentées d'autres entrées non vues au cours de l'apprentissage. Nous avons utilisé Matlab dans le code de calcul avec la boîte à outils Netlab [9] auquel l'algorithme choisi est le "Scaled Conjugate Gradient" avec les remarques faites dans [10].

Pour apprécier les performances des modèles, on a fait appel au critère $R^{2}$ très utilisé en hydrologie défini par [11].

$$
R^{2}=1-F^{2} / F_{0}^{2}=1-\frac{\sum_{i=1}^{n}\left(Q_{o b s, i}-Q_{c a l c, i}\right)^{2}}{\sum_{i=1}^{n}\left(Q_{o b s, i}-\overline{Q_{o b s}}\right)^{2}}
$$

Ce critère variant dans l'intervalle]- $\infty, 1]$ a l'avantage d'être facile à interpréter. II estime l'amélioration d'ajustement que l'on obtient en utilisant le modèle pour simuler les débits par rapport à un modèle 'zéro' (modèle de référence) qui donnerait sur toute la période considérée un débit constant égal au débit moyen.

Après avoir sélectionné les modèles jugés les plus fiables avec les bases test annuelles complètes, on les teste avec une base seulement composée de 30 jours de données continues disponibles. La base test est alors construite au fur et à mesure avec les données pluviométriques moyennes journalières de deux années et la hauteur de la rivière obtenue avec la prédiction. La hauteur de la rivière prédite est alors supposée être la hauteur moyenne journalière au cours de la période de deux années considérées.

Pour avoir le modèle global la technique de validation croisée en $k$-blocks est appliquée. La méthode consiste à faire l'apprentissage sur $k-1$ blocks et le test sur le $k^{\grave{m} m e}$, ce dernier variant d'une fois à l'autre. Cela conduit à avoir $k$ phases d'apprentissage et de test, où l'ensemble de test parcourt l'ensemble des données supervisées disponibles.

\section{2-2. Les données et lieu d'étude}


La base de données utilisée est obtenue avec la contribution du service de la hydrologie de Madagascar par le biais de l'Autorité pour la Protection contre les Inondations de la Plaine d'Antananarivo (APIPA), l'organisme qui se charge de la prévention de l'inondation dans le bassin de l'lkopa. Ces données couvrent les années 2001 à 2004.

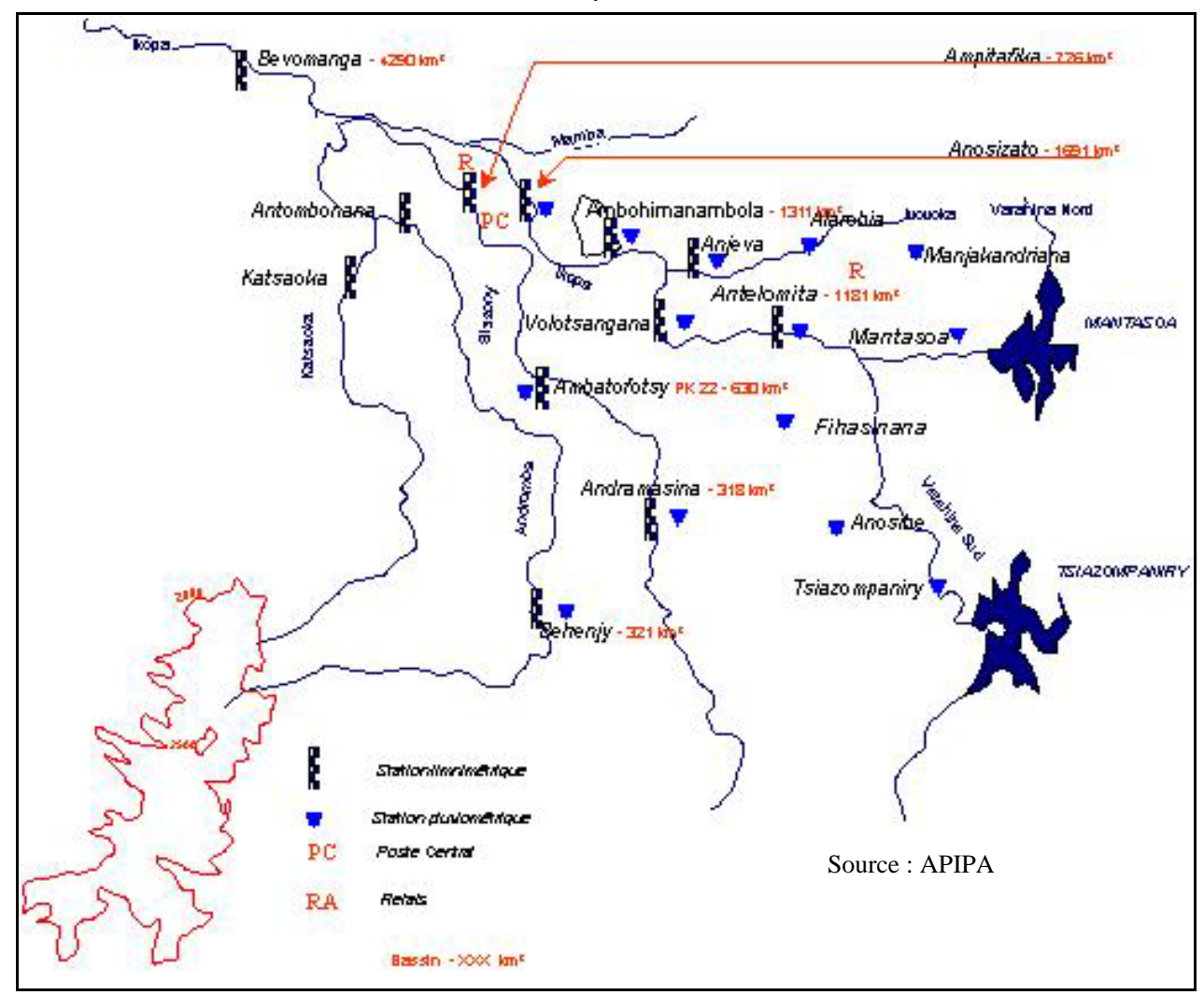

Figure 2: Réseau d'annonce de crues d'Antananarivo

L'APIPA dispose d'équipement d'annonce de crues qui est composé de 18 stations de mesure automatique de pluie et / ou de débit dans le bassin versant de l'lkopa en amont de Bevomanga (4 $300 \mathrm{~km} 2$ ). (e qui nous intéresse sont les affluents en amont qui se trouvent dans les bassins plus petits et inférieurs à $300 \mathrm{~km}^{2}$ pour un aménagement des barrages en vue de la production pico ou micro hydroélectrique. La carte de localisation des stations de recueil de mesure des hauteurs d'eau sur les affluents de l'Ikopa est présentée à la Figure 2.

Seules les stations pourvues en même temps de mesures des pluie et débit ou hauteur limnimétrique qui nous intéressent pour notre modélisation pluie-débit. 
Ainsi les cinq stations à savoir Ambohimanambola, Anjeva, Volotsangana, Andramasina et Ambatofotsy sont retenues.

Les données horaires à la disposition sont converties en données journalières. Nous avons pris la hauteur limnimétrique seulement à une heure fixe de la journée à cause de la recommandation en hydrologie [12] et la pluie recueillie pendant les 24 heures avant. Pour notre cas cette heure est fixée à 18 heures. Les hautes terres et le versant Est de Madagascar représentent des similarités en terme de géologie et de pluviométrie [13], ce qui justifie le choix du lieu d'étude.

\section{Résultats et discussion}

Les critères de Nash enregistrés pour les 5 bases test sont groupés dans le Tableau 2.

Tableau 2 : Nash pour les 5 bases test

\begin{tabular}{||l|l||}
\hline BASE TEST & NASH \\
\hline Volotsangana & 0.75 \\
\hline Ambohimanambola & 0.84 \\
\hline Anjeva & 0.53 \\
\hline Andramasina & 0.66 \\
\hline Ambatofotsy & 0.70 \\
\hline
\end{tabular}

Les modèles sont calés pour une base de test qui est complète de 365 jours de l'année. Le critère de Nash minimum observé est de 0,53 . Ce résultat est obtenu à la station d'Anjeva pendant l'année 2003 (Figure 3ત). Pour la station d'Ambohimanambola ce critère est de 0,84 . Les petites rivières ont la particularité de présenter des fluctuations très fréquentes. En effet leur réponse à la sollicitation de la pluie est plus rapide que les grands cours d'eau.

Cette faible performance pour Anjeva est provoquée par l'existence des pics extraordinaires aux alentours de $340^{\text {ème }}$ jour que nous voyons encore sur la Figure 4a malgré le calcul de la moyenne entre 2002 et 2003.

La Figure 4 montre les résultats de prévision de hauteur moyenne journalière entre 2002 et 2003 avec seulement des données de mesure de 30 jours. Puis la base de test est complétée au fur et à mesure en hauteur de rivière prédite avec les données pluviométriques moyennes des deux années. La hauteur de la rivière prédite est 
alors supposée être la hauteur moyenne journalière au cours d'une période de deux années considérées.

Les critères de Nash enregistré sur Anjeva et Ambohimanambola sont respectivement de 0,10 et 0,46 . II s'agit de la présentation de résultats extrêmes enregistrés au niveau de deux bassins. La baisse de la performance est prévisible mais ces résultats obtenus montre que les modèles représentent le régime annuel des cours d'eau nettement mieux que le débit moyen annuel. Les hauteurs représentées dans la Figure 4 sont supposées être les hauteurs moyennes journalières pendant les deux années de données disponibles.
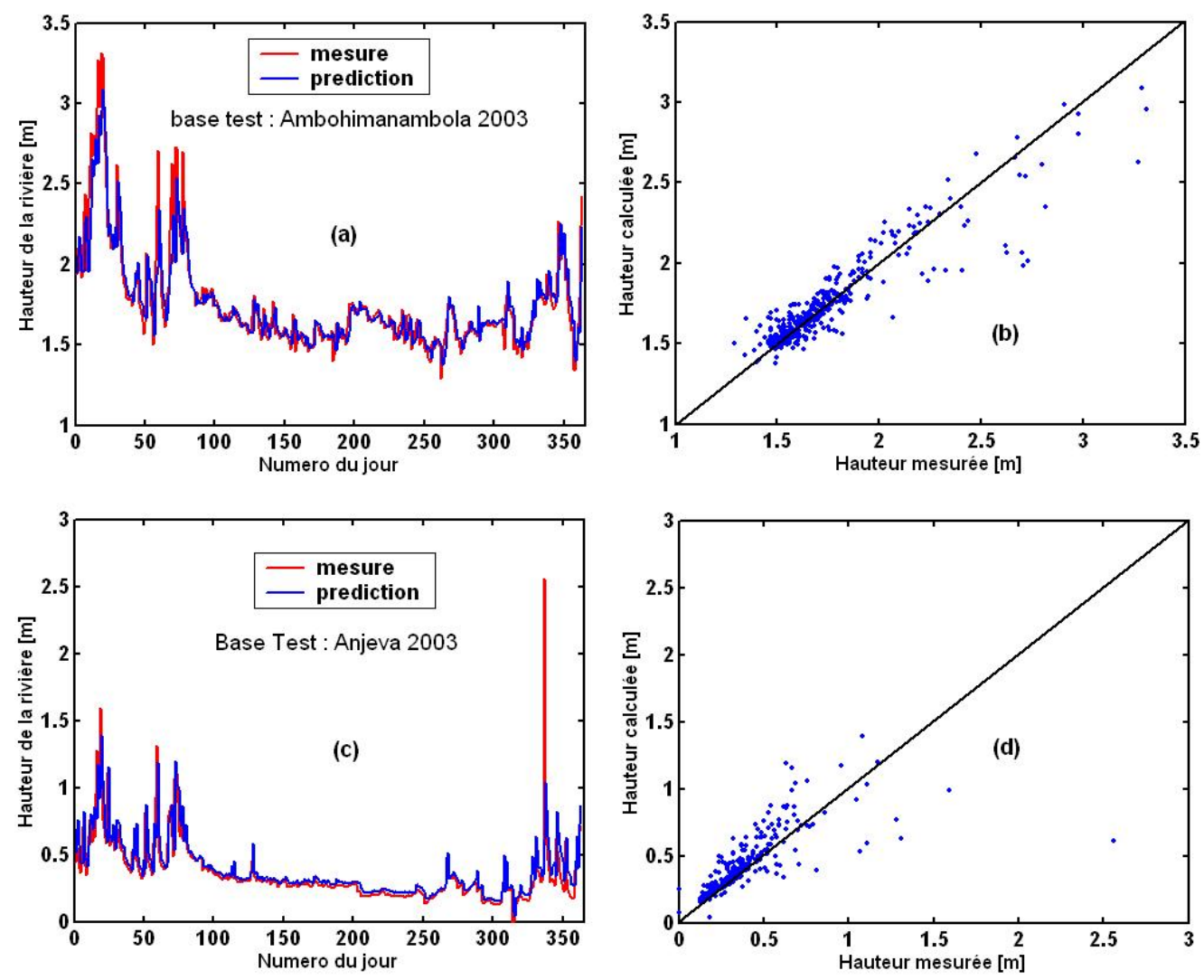

Figure 3 : Régime au cours de l'année 2003 pour la station d'Anjeva et d'Ambohimanambola, erreurs de sortie

La présence de la saisonnalité est toujours marquée dans tous les cas. La fluctuation des courbes est due au fait que la prédiction journalière est dictée par la pluie. La 
pluviométrie présente toujours des sauts même si on a effectué la moyenne. La moyenne de deux années n'arrive pas à lisser la pluviométrie.

Pour une exploitation en conversion hydroélectrique, la courbe de débits classés définie comme la courbe donnant l'historique des débits moyens quotidiens d'un site, classés en fonction du pourcentage du temps où le débit est supérieur ou égal à ce débit, est proposée. La relation entre le débit et la hauteur d'eau est purement algébrique qu'il nous est permis d'utiliser directement cette dernière (Figure 4.b,d).
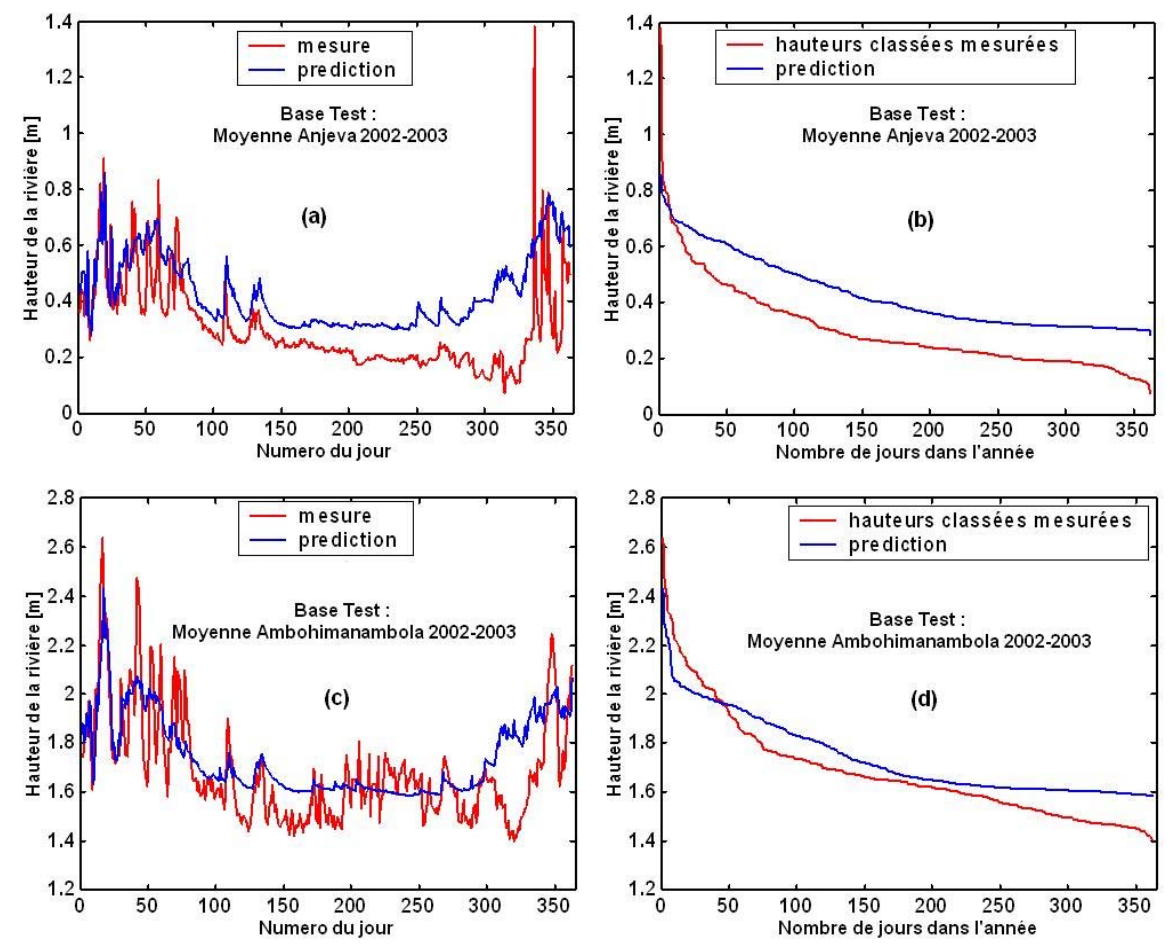

Figure 4 : Moyenne journalière au cours de 2002-2003 pour la station d'Anjeva et d'Ambohimanambola ; hauteurs classées

On peut dire que la moyenne de deux années ne suffit pas pour lisser les courbes. Mais déjà sur les deux années, l'exploitation des courbes de hauteurs classées pour l'évaluation en potentiel hydroélectrique est plus rassurante en terme de rendement par rapport à l'utilisation tout simple de débit minimum d'étiage de l'année de mesure. En effet le débit minimum change selon que l'année soit excédentaire ou déficitaire en pluviométrie. Alors que d'après les courbes, la hauteur de rivière assurée pendant 300 jours est plus proche de la prédiction que de la hauteur 
minimale. L'existence des données pluviométriques de plusieurs années donne plus d'assurance à la courbe des hauteurs classées obtenues par le modèle.

\section{Conclusion}

On peut conclure que les Réseaux de Neurones Artificiels représentent des outils très exploitables pour les modèles globaux de pluies-débits dans le but de combler les déficits en termes de données de relevés. Néanmoins il est toujours affirmé que plus les données sont nombreuses plus les prédictions approchent les valeurs réelles. Dans notre cas les seules données de deux années présentent encore beaucoup de fluctuations.

Les résultats sont obtenus avec la structure la plus simple et les algorithmes d'apprentissage sont à améliorer pour des meilleures efficacités. II est aussi intéressant de revoir le cas du nombre des variables en entrée et de vérifier par l'analyse de sensibilité leur pertinence dans le modèle.

\section{Références}

[1] - Ministère des Finances et de l'Economie /INSTAT/UE, 2006, Enquête Périodique auprès des Ménages 2004 : Appui à la mise en place d'un système national intégré de suivi de la pauvreté.

[2] - L. Monition, M. Le Nir ef J. Roux, Les Micro Centrales Hydroélectriques, Edition Masson, Paris (1981).

[3] - P. Coulibaly, F. Anctil et B. Bobée, Prévision hydrologique par réseaux de neurones artificiels : état de l'art, Can. J. Civ. Eng, 26 (1999) 293-304.

[4] - M.P. Rajurkar, U.C. Kothyari and U.C. Chaube, Modeling of the daily rainfall-runoff relation ship with artificial neural network, Journal of Hydrology, 285 (2004) 96-113.

[5] - A.Y. Shamseldin, Application of a neural network technique to rainfall-runoff modelling, Journal of Hydrology, 199 (1997) 272-294.

[6] - W.S. McCulloch and W. Pitts, A logical calculus of the ideas immanent in nervous activity, Bulletin of Mathematical Biophysics, 5(1943), 115-133.

[7] - K. Hornik, M. Stinchcombe and H. White, Multilayer feedforward networks are universal approximators, Neural Networks, 2(5) (1989), 359-366.

[8] - D.E. Rumelhart, G.E. Hinton, R.J. Williams, Learning internal representations by error propagation, Parallel Distributed Processing, 1(1986) 318-362. 
[9] - I.T. Nabney, Netlab: Algorithms for Pattern Recognition, Springer-Verlag, London (2002)

[10] - Y.M. Chiang, L.C. Chang and F.J. Chang, Comparison of static-feedforward and dynamic-feedback neural networks for rainfall-runoff modelling, Journal of Hydrology, 290 (2004) 297-311

[11] - J.E. Nash and J.V. Sutcliffe, River flow forecasting through conceptual models, Part L—a discussion of principles, Journal of Hydrology, 10(1970) 282-290.

[12] - World Meteorological Organisation, Intercomparison of Conceptual Models Used in Operational Hydrological Forecasting, Rapport d'hydrologie opérationnelle $\mathrm{N}^{\circ}$ $7,0 M M-N^{\circ} 429$, Genève (1987).

[13] - P. Chaperon, J. Danloux, L. Ferry, Fleuves et Rivières de Madagascar, Editions IRD, Paris (1993). 